\section{I-STAT: An interactive statistical program package*}

\section{LORNE ROSENBLOOD and KENNETH CHAPMAN Enversitn of Victoria. Victoria, B.C.. Canada}

This is a package of general purpose interactive statistical programs. The package is ideal for students or researchers wishing to do statistical analyses. The programs provide useful feedback for those who wish an immediate answer to their statistical problem. No programming knowledge is required. The program selects and controls the execution of the routines needed according to the responses of the user. The programs have been carefully designed to ask the user, in simple English, all questions necessary to do the analysis. If the user encounters difficulties with a program or is unfamiliar with the particular program, he can request detailed explanations, including examples. The programs have been written so a user familiar with them can bypass the self-contained explanations. All data entered are checked for validity, and the user is instructed to correct any errors. Data can be saved for immediate input to other programs. The output is arranged with variable and factor names for easy readability and understanding of the results. There are presently six programs available.

\section{PROGRAMS}

(1) Frequency count and statistics. This program takes raw data. The output is a frequency count, sample size, maximum and minimum values, range, mean, variance, standard deviation, mean deviation, median, and modes (if any).

(2) Analysis of variance for the equal-n case. This program accepts up to five factors with any number of levels and any number of Ss. The output is a standard analysis of variance table, fully labeled, with exact probability levels for each test.

(3) Contingency tables and chi-squared calculations. This program takes raw data and tabulates it according to partition values entered. The output is a fully labeled contingency table, chi-square, and the probability level. The program will also accept a contingency table for input.

(4) Correlation matrix generation. This program calculates the means, variance, and correlation matrix from the raw data with any number of variables and any number of Ss. The output is clearly labeled for easy interpretation.

(5) Multiple regression analysis. This program accepts any number of predictors and Ss. The output is a fully labeled regression analysis including, if desired, simple or partial correlations, tests on the regression and residuals, and the regression values in tabular form.

(6) Random number generators. A set of programs provides selection of integers with or without replacement from a specified set, or random telephone numbers can be generated from a specified set of seven-digit numbers.

\section{LANGUAGE}

The routines are written in York APL but can be easily modified to IBM's APL 360 . The programs were developed on an IBM System/370 Model 155 running under OS(MVT) using 2741 communications terminal.

\section{RESTRICTIONS}

The amount of data that can be worked with is dependent upon the size of the work area provided by the user's system. With a workspace of $30 \mathrm{~K}$ bytes, analysis can be done on $100 \mathrm{Ss}$, with 10 variables per $S$.

\section{AV AIL ABILITY}

Free listings for the routines can be obtained from Lorne Rosenblood, Department of Psychology, University of Victoria, Victoria, B.C., Canada.

\section{REFERENCE}

Smillie, K. W. Statpact 2: An APL statistical package. 2nd Ed. Alberta, Canada: Department of Computing Science, University of Alberta, June 1969.

(Received for publication November 16, 1973; accepted November 23,1973.)

\section{DICORR: A FORTRAN program for} computing dichotomous correlation coefficients

\author{
JOHN L. KOREY \\ Department of Political Science \\ California State Polytechnic University \\ Pomona, California 91768
}

DICORR computes a matrix of correlation coefficients among dichotomous variables. Any of the following coefficients may be selected: Yule's Q, Yule's $\mathrm{Y}$, phi, phi/phi max. Input data must be coded in single-column integer format. Output for the program includes: (1) list of options selected by user (2) summary of responses for each variable; (3) printout of correlation matrix; (4) punched output of correlation matrix (optional).

\section{COMPUTER}

The program has been run on both the CDC 3150 and the CDC 3300

\section{LANGUAGE}

The program uses FORTRAN IV. It has been run under both ANSI (i.e., USASI) and MS FORTRAN compilers.

\section{LIMIT ATIONS}

The program will handle any number of cases. It is presently dimensioned to handle up to 50 variables. Depending upon the amount of core storage available to the user, program dimensions may be adjusted simply by changing the DIMENSION statement at the beginning of the program.

\section{ACKNOWLEDGMENT}

The program was developed at California State Polytechnic University, Pomona, and computer time was made available by the institution.

\section{AVAILABILITY}

A source listing, write-up, and sample run may be obtained free of charge by writing the author. 